\title{
Plastic Optical Fibre Sensors for Structural Health Monitoring: A Review of Recent Progress
}

\author{
K. S. C. Kuang, ${ }^{1}$ S. T. Quek, ${ }^{1}$ C. G. Koh, ${ }^{1}$ W. J. Cantwell, ${ }^{2}$ and P. J. Scully ${ }^{3}$ \\ ${ }^{1}$ Department of Civil Engineering, National University of Singapore, Block E1A, \#07-03, 1 Engineering Drive 2, Singapore 177576 \\ ${ }^{2}$ Department of Engineering, University of Liverpool, Brownlow Hill, L69 $3 \mathrm{GH}$ Liverpool, UK \\ ${ }^{3}$ The Photon Science Institute, University of Manchester, Oxford Road, M13 9PL Manchester, UK
}

Correspondence should be addressed to K. S. C. Kuang, cveksck@nus.edu.sg

Received 4 March 2009; Revised 24 June 2009; Accepted 30 July 2009

Recommended by Christos Riziotis

\begin{abstract}
While a number of literature reviews have been published in recent times on the applications of optical fibre sensors in smart structures research, these have mainly focused on the use of conventional glass-based fibres. The availability of inexpensive, rugged, and large-core plastic-based optical fibres has resulted in growing interest amongst researchers in their use as low-cost sensors in a variety of areas including chemical sensing, biomedicine, and the measurement of a range of physical parameters. The sensing principles used in plastic optical fibres are often similar to those developed in glass-based fibres, but the advantages associated with plastic fibres render them attractive as an alternative to conventional glass fibres, and their ability to detect and measure physical parameters such as strain, stress, load, temperature, displacement, and pressure makes them suitable for structural health monitoring (SHM) applications. Increasingly their applications as sensors in the field of structural engineering are being studied and reported in literature. This article will provide a concise review of the applications of plastic optical fibre sensors for monitoring the integrity of engineering structures in the context of SHM.
\end{abstract}

Copyright $\odot 2009$ K. S. C. Kuang et al. This is an open access article distributed under the Creative Commons Attribution License, which permits unrestricted use, distribution, and reproduction in any medium, provided the original work is properly cited.

\section{Introduction}

In recent years, structural health monitoring has attracted significant interest from academia, government agencies, and industries involved in a diverse field of disciplines including civil, marine, mechanical, military, aerospace, power generation, offshore and oil and gas. The aim of SHM is to detect damage initiation and subsequently monitor the development of this damage using structurally-integrated sensors in order to provide early warning and other useful information for successful intervention to preserve the structural integrity of the host. A number of commonly monitored parameters used for SHM applications include the detection or measurement of strain, load, displacement, impact, $\mathrm{pH}$-level, moisture, crack width, vibration signatures, and presence of cracks.

Over the last two decades, optical fibre sensors have attracted substantial attention and shown to be capable of monitoring a wide range of physical measurands for SHM applications. The advantages of optical fibre sensing in engineering structures are well known and these include their insensitivity to electromagnetic radiation (especially in the vicinity of power generators in construction sites), being spark-free, intrinsically safe, non-conductive and lightweight, and also their suitability for embedding into structures. To date, a number of key optical fibre sensors have been reported and their applications for damage detection in composite structures are given in review articles elsewhere $[1,2]$. Optical fibre-based sensors such as fibre Bragg gratings (FBG), intensiometric and polarimetric-type sensors and those based on interferometric principles (e.g., Fabry-Perot) have been shown to offer specific advantages in their niche area of applications.

Of the various types of optical fibre sensors, intensiometric sensors represents one of the earliest and perhaps the most direct and basic type of optical fibre sensor used for SHM purposes [1]. Here, the sensing principle is straightforward and relies on monitoring the intensity level of the optical signal as it modulates in response to the measured quantity. Although monitoring of the intensity level of optical signal 
has often been cited to be a drawback as a result of possible power fluctuation in the signal level and influence of external environment unrelated to the measured parameter (e.g., micro and macro bending along the fibre length), standard referencing techniques may be used to counter this problem. With the availability of stable and inexpensive light sources and low bend-sensitivity fibres, the intensitybased approach offers excellent commercial prospect for large-scale applications from a cost-effectiveness point of view. In addition, the intensity-based technique is also suitable for frequency analysis in vibration measurements since precise and absolute measurement of the structural strain or displacement values are not required-given that the sensor has sufficient sensitivity to detect the oscillatory nature of the vibration signal.

Plastic optical fibres (POFs) with their large core sizes (diameters ranging typically from $0.25 \mathrm{~mm}$ to $1 \mathrm{~mm}$ are readily available) and high numerical apertures (0.47) lend themselves well to be used as intensity-based optical fibres sensors. Indeed, many of the POF sensors developed and demonstrated for a variety of SHM applications in recent times are based on intensity modulation using these multimode fibres [3-16]. The core of the fibre could be made from polymethymethacrylate (PMMA), polycarbonate (PC), polystyrene (PS) and more recently cyclic transparent optical polymer (CYTOP), which offers the lowest attenuation of $50 \mathrm{~dB} / \mathrm{km}$ at $650 \mathrm{~nm}$ compared to $160 \mathrm{~dB} / \mathrm{km}$ for PMMAbased POF. The cladding layer of the fibre is generally made of fluorinated polymers. At present, most POF sensors are step-index PMMA-based due to their wider availability and lower cost. Other variants of POFs including multicore fibres, double-step-index fibres, multi-step-index fibres and graded-index fibres have also been introduced to improve the bandwidth and to lower the bending sensitivities (by means of multiple smaller cores and optimising on the refractive index profile). Single-mode POFs are presently obtainable commercially (e.g., Paradigm Optics Inc.) although their availability is still limited worldwide. Bragg gratings, which are commonly applied to single-mode silica fibres using ultra violet laser light to create the interference pattern to induce periodic changes in refractive index of the core, have also been demonstrated on doped plastic optical fibres and undoped bulk PMMA in recent years [17-19]. More recently, micro-structured POF have been introduced and these have received significant attention as a promising class of fibre for new sensor applications [20, 21], achieving unique optical properties via a pattern of holes down the full length of the fibre. Optical properties include enabling single-mode fibre to be made from a single matrix material with characteristics controlled by photonic bandgap effects. Unlike single mode POF, single-mode microstructured POF has a visible loss of around $1 \mathrm{~dB} / \mathrm{m}$ and are single moded for wide (theoretically endless) range of wavelengths. Bragg gratings [22] and long period gratings [23] have been created within mPOF. Advantages include the possibility of optimising the sensitivities of the different loss features to a range of measurands by adjusting the hole geometry, and using asymmetric microstructures for directional bend sensitivity [24].
In addition to being cheaper than their glass-counterpart, plastic fibres offer better fracture resistance and flexibility compared to bare glass fibres. They also offer ease of termination, safe disposability and ease of handling. It has been reported that plastic optical fibre has an elastic limit of $10 \%$ compared to $1 \%$ in silica and can withstand strains more than 30\% without breakage [25] — this could be a significant benefit for structural health monitoring applications involving large strains greater than that measurable by glass-based fibre sensors. For monitoring internal parameters of a structure, for example, when it becomes desirable to embed sensors within concrete structures, POF sensors offer a possible solution since the extremely alkaline $(\mathrm{pH}$ 12) environment of the concrete mixture is known to be corrosive to standard glass fibres [26]. Also, the presence of moisture can weaken the glass core and accelerates crack growth in the fibre. For glass-based sensor, although a polymer coating may be applied in order to protect the glass fibre from the corrosive environment, this will incur additional cost. Finally, glass-based optical fibre sensors are fragile and in general not amenable to rough handling and are highly susceptible to fracture in harsh engineering environment. In view of the advantages associated with plastic optical fibres, intensive research is underway to assess their potential for smart structure and structural health monitoring applications.

\section{Recent Development in POF Sensors for SHM}

2.1. Intensity-Based POF Sensors for SHM. The ease of monitoring the light intensity level in these large core fibres (typically $1 \mathrm{~mm}$ step-index multi-mode type) naturally leads to their development as intensity-based sensors. The availability of in-expensive solid-state light emitters and detectors allows the POF sensors to be conveniently integrated to external set-ups such as control and data acquisition systems. Indeed, the simplicity in design associated with intensiometric measurements has resulted in the various applications of POF sensors not only for SHM but for a variety of other sensing applications [27-29]. POF sensors were demonstrated to have the capability to measure parameters such as strain, curvature, bending displacement as well as for detecting cracks within the structure subjected to either quasistatic or dynamic loading [3-16].

In general two classes of intensity-based sensing have been reported and they are grouped based on whether the optical fibre is an intrinsic or extrinsic sensor. In an intrinsic sensor, modulation of the optical signal is a direct result of the physical change in the optical fibre in response to some measurands (e.g., signal change due to the micro- or macrobending of the fibre). On the other hand, in an extrinsic optical fibre, the signal modulation takes place outside the optical fibre (e.g., signal change due to the changing gap distance between two cleaved fibre surfaces).

Kuang et al. [3] investigated the use of a low-cost, intensity-based intrinsic POF sensor for monitoring the mechanical response of a number of plastic specimens. In their study, the plastic fibre used (ESKA CK40) was 


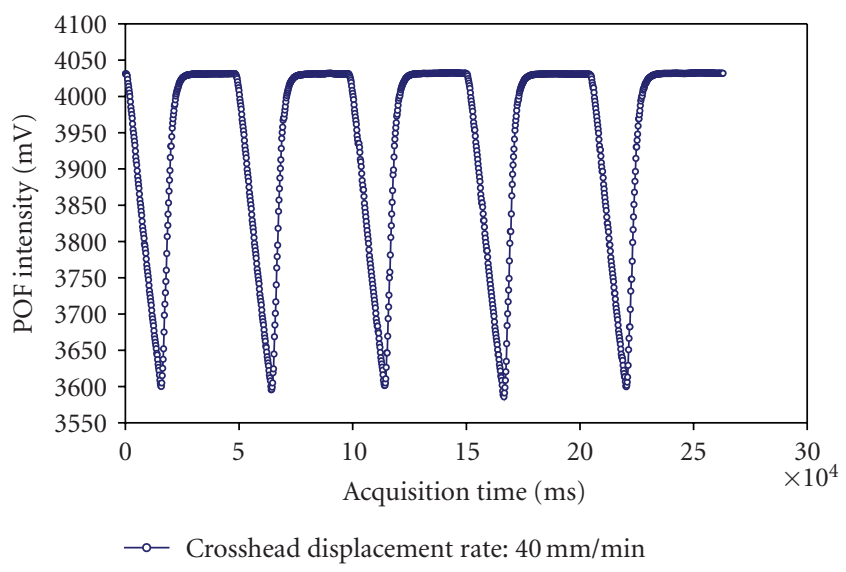

(a)

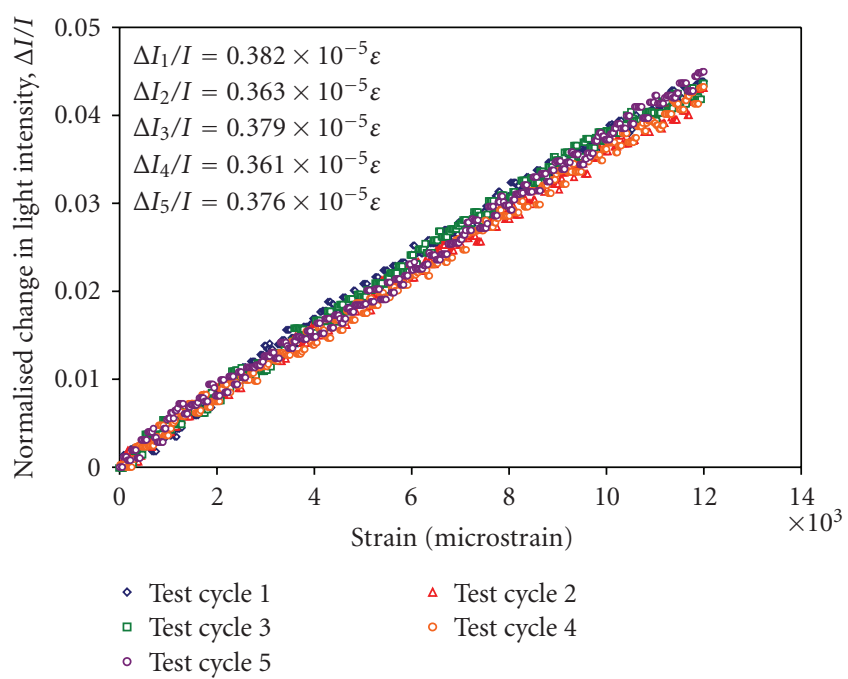

(b)

FIgure 1: (a) Plot of the POF sensor response under cyclic flexural loading highlighting the stability and responsiveness of the sensor (after [3]). (b) Plot showing the POF sensor signal during a series of tensile tests (after [3]).

a $1 \mathrm{~mm}$ diameter multimode step-index type supplied by Mitsubishi Rayon Co. Ltd. By removing a segment of the POF's core and cladding layer over a pre-determined length, the aim was to promote light loss in this region due to reduction in the number of modes undergoing total internal reflection when the fibre was bent. The sensitised region (ranging from $70 \mathrm{~mm}$ for smaller specimens to $300 \mathrm{~mm}$ for larger ones) was noted to possess directional sensitivity and hence important to ensure the relative planar orientation of the segment of the POF sensor and the direction of loading. The study demonstrated that the POF sensor used exhibited high responsiveness to bending (strain-normalised optical loss coefficient of approximately $1.8 \times 10^{-5} / \mu \varepsilon$ ) and could be configured to render it sensitive to in-plane axial loads by simply curving the sensing region of the POF in the appropriate orientation with respect to the direction in which the strain is to be measured. Figure 1(a) shows the signal of POF sensor under a cyclical flexural load

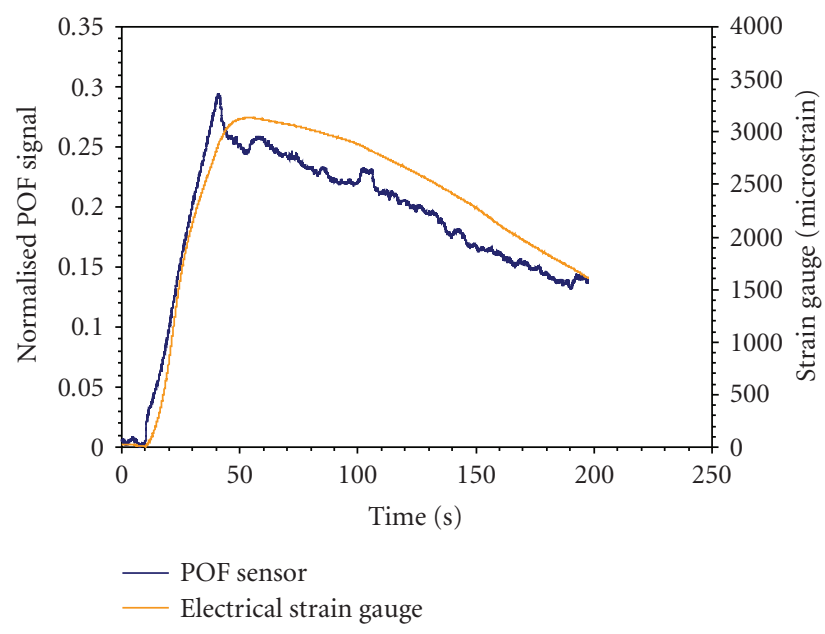

FIGURE 2: Plot showing the response of the POF sensor following activation of the Ni-Ti sheet (after [34]).

while Figure 1(b) illustrates the repeatability of the sensor configured for tensile strain measurement. Although the tests stopped at bending and tension strains of $0.7 \%$ and $1.2 \%$ respectively, the ability of the POF to measure higher strain values was expected to be achievable. Strain values up to $15.8 \%$ have been reported in a single-mode POF by Kiesel et al. $[30,31]$ while other studies using standard POF have measured strains up to $45 \%$, although it was noted that depending on strain rate and temperature, the fibre could endure more than $80 \%$ strain $[32,33]$.

The potential use of POF for SHM purposes was also investigated for the dynamic monitoring ability in fibre composites $[9,10]$. The upper limit of the frequency tested was $30 \mathrm{~Hz}$ (limited by the motor used). Here a POF sensor, identical in terms of theoretical background and operating principle to Kuang et al. [3], was attached to a cantilever-type composite beam to monitor the free vibration of undamaged and damaged specimens following low-velocity impacts. The sensitised POF sensors used was sufficiently sensitive to monitor the change in the damping ratio to characterize the reduction in postimpact flexural modulus and residual strengths of a composite beam with increasing level of impact damage. In the experiment, the POF sensor was able to detect a change in the damping ratio as small as $2.5 \%$. In a later study [34], the POF sensor was applied to a nickel-titanium fibre metal laminate to monitor the morphing response of the hybrid laminate and the POF signal was found to agree well with collocated electrical strain gauges. Following activation of the smart fibre metal laminate (FML) by air through a heat gun, the shape memory alloy (SMA) layers deformed according to the shape it was trained (i.e., curved) and the POF sensor was found to faithfully monitor the flexural response of the smart composite. Figure 2 shows the response of the POF compared to the strain gauge reading.

Further work on the use of an extrinsic POF sensor to monitor the deflection of a smart composite was done by Kuang et al. [35]. The operating principle was straightforward-the sensor relied on the monitoring of the 


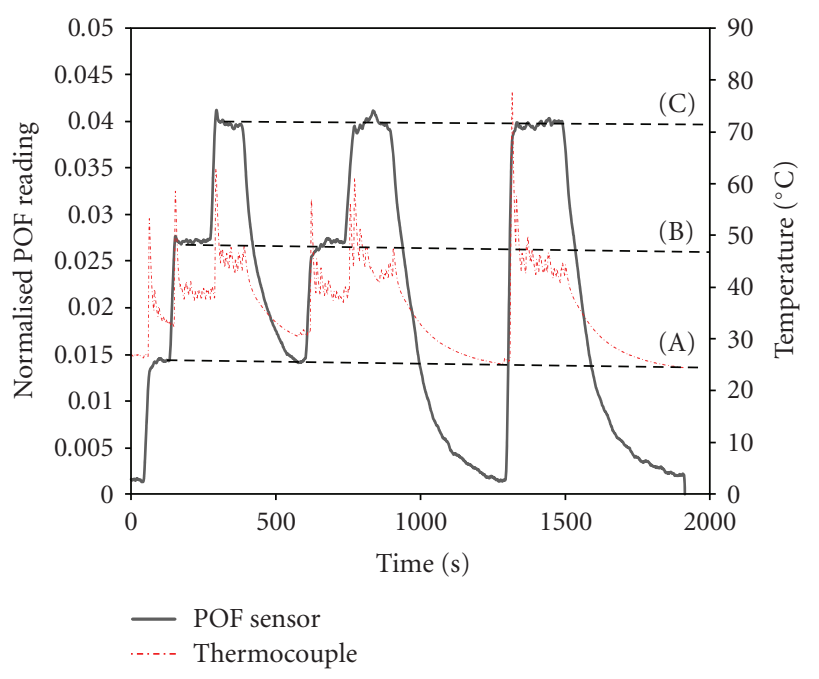

FIgUre 3: Plot showing the response of the POF sensor highlighting the successful use of the POF data in the control loop to achieve the amount of deflection desired (after [35]).

optical power transmitted through an air-gap between two cleaved optical fibre surfaces. The two fibres were aligned within a housing in which the fibres could slide smoothly. The gap between the cleaved surfaces changes in proportion to the applied strain resulting in the increase or decrease of the transmitted power. A standard red LED $(650 \mathrm{~nm})$ and photodiode were used to illuminate and monitor the optical power during the test. Here, thin-film heating technology was introduced to assess their potential for their integration into the smart FML for SMA activation. To provide the sensor feedback signal, the POF sensor was attached to the smart composite allowing the deflection or morphing response of the specimen to be accurately controlled. The POF sensor used here was modified based on a design described in an earlier work [15]. The desired amount of deflection of the beam was pre-set using a controller and the POF sensor reading was used as a feedback signal to achieve the desired deflection. The deflection of the beam specimen was monitored continuously via a data acquisition set-up which logged the POF sensor output simultaneously. Figure 3 shows the data of the POF sensor readings at three different FML beam deflections (A) to (C). The result shows that the FML beam could be controlled accurately to within $3 \%$ of the desired deflection using the POF feedback data with very little overshoot.

Another variation of the intensity-based POF sensor for monitoring structural displacement was proposed by Babchenko et al. [4] based on the bending of a multi-looped POF that has sensitised multistructural imperfections on the outer side of its core. The structural imperfections were created on the outer side of the fibre's core by abrading the fibre surface. The imperfections were created in the form of small scratches perpendicular to the curve plane similar in concept to earlier studies $[3,7,10]$. The increased loss of light at the sensitised region due microbending was then related to the amount of displacement. The study used a simple mechanical set-up where the fibre sensor was located between a top and bottom plate connected to a micrometer allowing the amount of bending of the fibre to be controlled. The authors argued that by adding more loops to the fibres and additional imperfections on the apex of the curve section of the POF, an inexpensive POF sensor could be created to monitor a variety of physical measurands, including strain, stress, vibration and pressure although the authors have not conducted any specific studies to show the actual performance of the proposed sensor to monitor the various loading conditions listed.

POF sensors have been applied to concrete structures in the field of civil engineering in view of their ruggedness and ease of handling compared to glass-based fibre sensors. Kuang et al. [11], conducted a series of flexural tests on scaled-specimens where POF sensors were attached to the bottom surface of the beam and showed that the sensors used were of sufficient sensitivity to detect the presence of hair-line cracks as illustrated in Figure 4. A crack width of approximately $0.04 \mathrm{~mm}$ was successfully detected using the POF sensor. In order to improve the sensitivity of the POF to beam deflection and crack initiation, a segment of the POF cross sectional profile was removed over a predetermined length $(7 \mathrm{~cm}$ for a series of scale-model specimens and $30 \mathrm{~cm}$ for full-scale specimens) by abrading the surface of the POF using a razor blade. In principle, the sensitisation process increases the loss in mode propagation when the fibre is bent. Exposing the fibre core by removing the cladding layer to create an evanescent field sensor is a well known technique commonly exploited for sensing purposes. Following tests on the scaled-specimen, the authors demonstrated the use of POF sensors to monitor the response of three-meter-long concrete beams subjected to a quasistatic lateral load in a three-point bend set-up. In their study, multimode stepindex plastic optical fibres were successfully applied to detect initial cracks in the beam and subsequently to monitor postcrack vertical deflection and finally to detect failure cracks in concrete beam. Figure 5 shows the plot summarising the results of the tests carried out for crack detection in concrete specimens.

In another study related to structural health monitoring of concrete beams, a liquid-filled extrinsic POF sensor design was employed to monitor the central deflection of a concrete specimen in a three-point bend configuration [14]. Here, four extrinsic POF sensors were used-each with a different liquid opacity injected into the housing cavity shown schematically in Figure 6(a). The principle of operation is the same as that described earlier where the transmitted optical power across a gap between two cleaved POF surfaces was monitored and related to the applied load or strain. Instead of an air-gap, the addition of an opaque liquid medium in the cavity of the housing increases the strain sensitivity of the POF sensor (up to approximately 25 times) as shown in Figure 6(b). Following the initiation of crack at the bottom surface of the concrete beam, the electrical strain gauge failed instantly while the POF was able to continue monitoring the response of the beam under the transverse load highlighting the advantage of the POF sensors over electrical strain gauges in this particular 

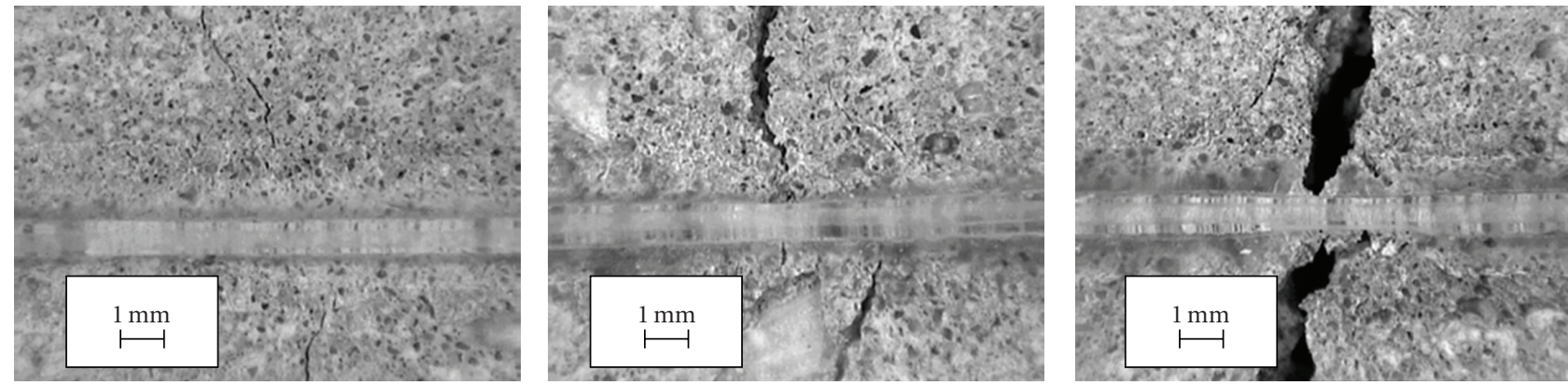

FIGURE 4: Photomicrographs showing the intersection of the crack in the crack specimens with the POF sensor (after [11]).

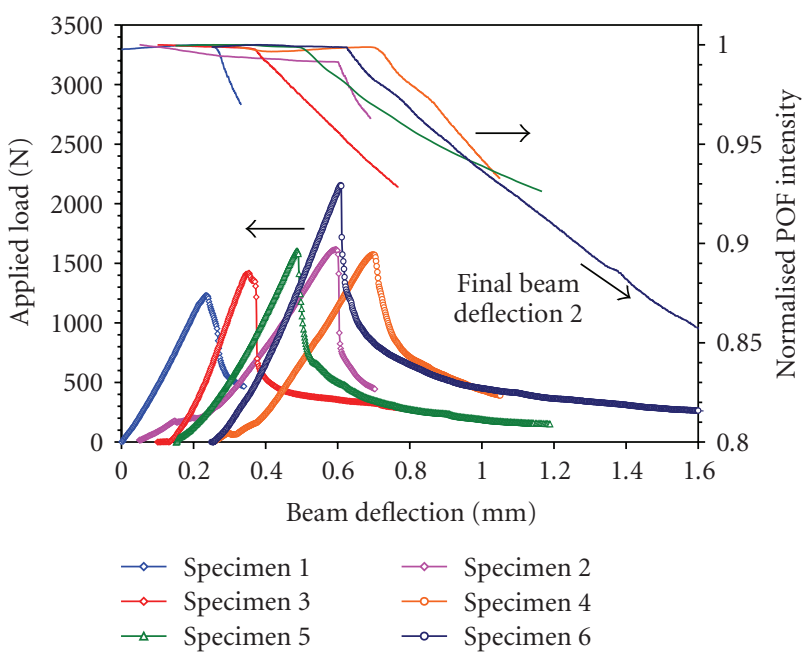

FIGURE 5: Summary of results for three-point bend tests on concrete specimens (beam deflections for specimens 2-6 have been offset for clarity of presentation) (after [11]).

application. As shown in Figures 7(a) and 7(b), the collocated electrical strain gauge was damaged at the first crack of the beam and was rendered useless limiting its usefulness for structural health monitoring purposes where surface cracks are frequently encountered. The POF sensors, however, did not appear to be significantly affected by the crack and were able to continue monitoring the loading process even after severe crack damage (crack width of approximately $2 \mathrm{~mm}$ and a corresponding POF strain of $4.7 \%$ ) has taken place in there steel reinforced beam specimen. Since the POF sensor was attached to the beam at the two points (which define its gauge length), the propagation of crack across the sensor has insignificant detrimental effect on its measurement capability.

In view of the potential of the POF sensor for vibration detection, it has also been used in another study on system identification for SHM in a composite beam using genetic algorithm as the parametric search method. An analysis using fast-Fourier transform of the acquired POF vibration signal compared well with a collocated piezofilm sensor and the result showed that the sensor was capable of detecting the shift in the various modal frequencies associated with

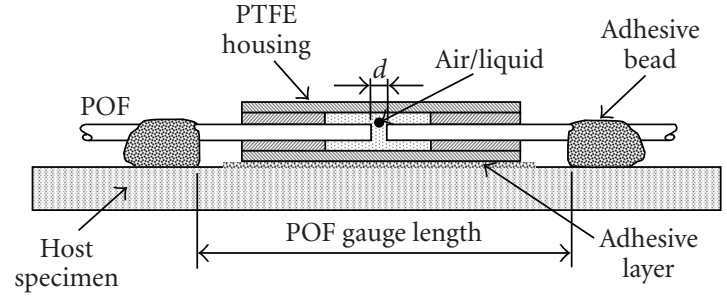

(a)

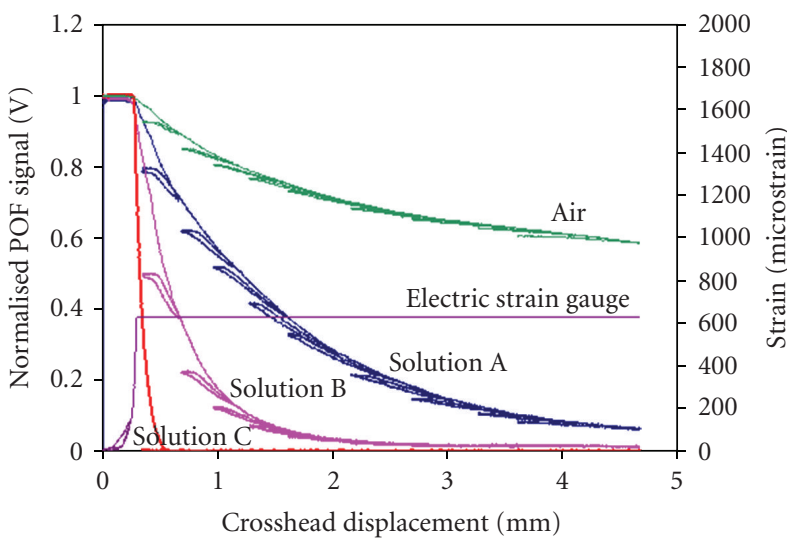

(b)

Figure 6: (a) Schematic of extrinsic POF sensor. (b) Plot showing the different POF sensor sensitivities corresponding to the different opacity of liquid used and the response of the electrical strain gauge attached to the bottom side of a concrete beam during a quasistatic cyclic test (after [14]).

different system characteristics or damage level [12-14]. The highest frequency detectable in the study was in excess of $1 \mathrm{kHz}$ [12] highlighting the potential of the system for vibration-based structural health monitoring.

In addition to concrete structures, POF sensors have been applied to monitor large strains (defined as greater than $10 \%$ ) developed in geotextile materials. Kuang et al. [36] reported that the intensity-based sensor used in their work could be customised to monitor strains as high as $40 \%$ or more. Based on a previous design [15], the cavity of the housing for this large strain sensor has been readapted and is shown schematically in Figure 8. The basic operating principle of the POF sensor used here relies on measuring 


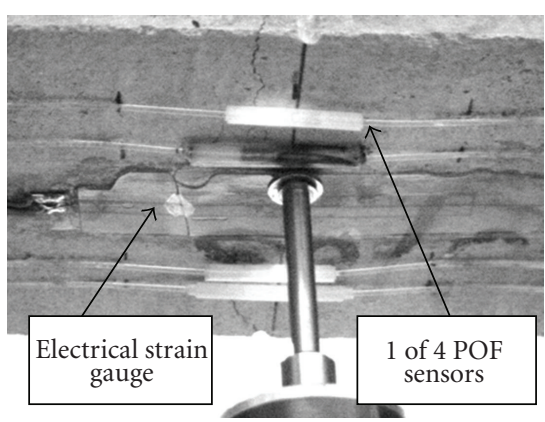

(a)

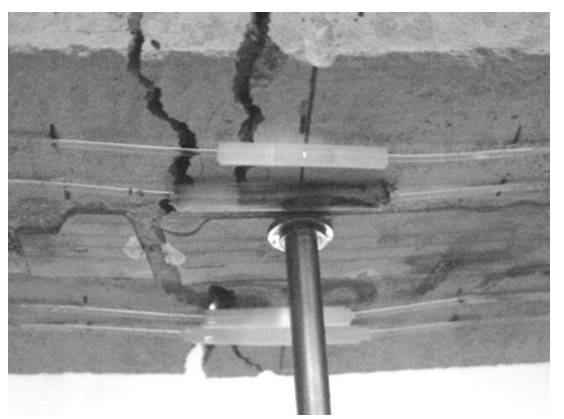

(b)

Figure 7: (a) Photograph showing the crack line across the electrical strain gauge at approximately $0.3 \mathrm{~mm}$ beam central deflection. (b) Photograph showing the widening of the crack after several loading cycles - the damage of the electrical strain gauge is evident while the POF sensors continued to monitor the loading process (after [14]).

the displacement of two cleaved fibre surfaces housed within the tube. Since the two ends of the POF were free to move under an applied axial load, the sensor strain measuring capability was not limited to the yield strain or elastic limit of the POF itself. The authors reported that the signal output of the sensor was directly related the separation of the two end faces and the sensor was initially calibrated with a linear variable displacement transducer before being attached to the geotextile host for strain measurement. POFs are available with protective polyethylene jackets and have a good resistance to damage under marine conditions and therefore are suitable candidates for a marine environment. Being inexpensive to produce and interrogate, it was proposed that the POF sensors were more cost-effective than other opticalbased sensors such as fibre Bragg grating (FBG) sensorsfor comparison, a POF sensor cost less than US\$1 while an acrylate-recoated FBG sensor would cost typically US\$100. More significantly, an FBG interrogator could range from US $\$ 15,000$ to US $\$ 40,000$ while in contrast, it is possible to fabricate an intensity-based system for under US\$200 using off-the-shelf parts highlighting the economic attractiveness of the proposed system.

POF has also been embedded in composite materials to monitor damage development. Takeda et al. [5, 6] utilised small diameter multimode POFs $(250 \mu \mathrm{m})$ for detection of matrix crack in advanced fibre composites. The POFs were

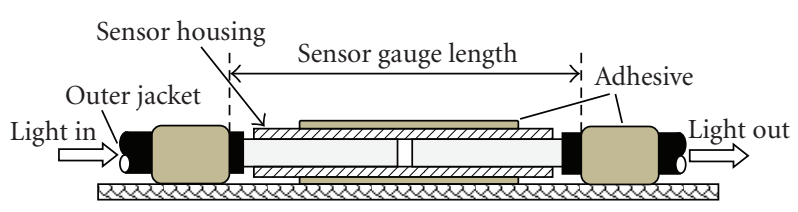

Geotextile substrate

FIGURE 8: Schematic of POF sensor and location of adhesives (after [36]).

embedded in both unidirectional and cross-ply composite lay-up. The sensors were subjected to the same curing cycle as the composites and the authors successfully demonstrated the possibility of embedding POFs into the fibre composites. This approach relies on changes in the optical power-strain relationship to infer damage. It was reported that when the unidirectional specimen was loaded in the axial direction, the optical power decreases linearly with strain while no damage was observed. In contrast, as a result of the transverse crack in the cross-ply laminate specimen, a non-linear optical response was noted following the initiation of cracks in the specimen. Takeda et al. contended that this observation supported their predicted response and hypothesised that the non-linearity observed in the optical response was an indication of local deformation of POF resulting from the damage in the host and hence demonstrated the viability of the technique for detection of transverse damage in composite materials.

Wong et al. [28] embedded a chemically-tapered POF in a carbon fibre composite to examine the potential for strain measurement. In the study, a POF with taper length of approximately $10 \mathrm{~mm}$ was used. Tapering a fibre forms a more sensitive evanescent field sensor because, as the fibre diameter decreases further with strain, the evanescent field penetrates further into the cladding rendering it sensitive to the applied strain. A series of tensile tests was conducted on a composite test beam with an embedded tapered POF sensor. The POF was embedded at the mid-plane of a four-ply woven carbon-fibre epoxy prepreg (Stesapreg EP121-C15-53). The composite lay-up was inserted in a picture frame mould and was processed at $75^{\circ} \mathrm{C}$ under 3 bar for 8 hours to allow curing of the epoxy matrix. The test specimen was subsequently examined and the POF sensor was found to be capable of withstanding the processing conditions. The authors showed that the embedded POF was capable of monitoring up to $1.4 \%$ strain with good repeatability.

Embedding of POF sensors in composite materials requires careful selection of the type of POF material since the processing temperatures vary according to the material system of the composites used. Although certain classes of POF such as polycarbonate and CYTOP are able to sustain higher processing temperatures compared to PMMA POFs without suffering significant optical and structural degradation, the range of composites which could be embedded with POF sensors is clearly limited to the maximum operating temperatures of the chosen POF.

Based on the literature reviewed, it is reasonable to conclude that intensity-based POF sensors offer a simple, 
in-expensive yet effective approach in monitoring specific aspect in structural health monitoring, in particular where high-resolution and precise measurements are not key requirements in the application. The concern over the fluctuation of optical intensity due to macrobending along the fibre or other perturbation not related to the measured quantity could be overcome with careful placement of the fibre (if possible) and referencing techniques and hence would not pose a significant barrier to its deployment. It has also been shown in the above review that, the apparent simplicity in sensor design, signal interrogation and acquisition, intensity-based monitoring approach using standard LEDs, photodetectors and low-cost data acquisition units allows users to implement a working system readily without the need for expensive equipment. The ease of handling large core intensity-based POF sensors outside the laboratory environment adds to its attractiveness for health monitoring of engineering structures. The review also provided examples of the use of intensity-based POF sensors for measurement of strain values less than a few hundred microstrains to that exceeding $40 \%$. The sensors were also demonstrated as surface bonded or embedded in structures. These reports highlight the versatility of the intensity-based POF sensors for measurement commonly performed in engineering applications and would indeed be the preferred technique in certain cases of SHM applications following careful consideration of its limitations and benefits.

\subsection{OTDR-Based POF Sensors for SHM. Distributed strain} sensing using a single fibre enables monitoring over a long section of structure and is highly desirable in structural health monitoring. Optical time-domain reflectrometry (OTDR) is a well known technique in telecommunication for fault analysis and has recently been applied to multi-mode standard POF for strain sensing applications. Despite large fibre core size and hence significant modal dispersion, some success was demonstrated for SHM applications. OTDR sensing exploits the monitoring of the backscatter light in an optical fibre following the launched of a short optical pulse at one end of the fibre. The backscatter signal is recorded as a function of time and then converted to distance measurement. Perturbations, such as strain or defects along the length of the fibre will result in either a peak reflection or loss in the backscatter signal at the location of the perturbation.

Husdi et al. and Nakamura et al. [37, 38] tested two types of PMMA-based multi-mode step-index fibres Eska Premier GH-4001-P and Super Eska SK-40 for the effect of mechanical deformation and temperature on the transmission and reflection properties of these fibres. A commercial photon counting OTDR system (with a position scale resolution of $10 \mathrm{~mm}$ ) for measuring the very weak backscattering light was used to interrogate the POF sensor. The study showed some interesting effects on the OTDR signal including the response due to (i) a flaw along a fibre (along a $300 \mathrm{~m}$ long fibre), (ii) a small bend (bending diameters ranging from $8 \mathrm{~mm}$ to $52 \mathrm{~mm}$ of a $300 \mathrm{~m}$ long fibre), (iii) transverse clamping (clamped over a $50 \mathrm{~mm}$ section at a distance $30 \mathrm{~m}$ from the input end), (iv) torsional strain (a section of $10 \mathrm{~mm}$ was twisted at a distance of $40 \mathrm{~m}$ from the input end along a $200 \mathrm{~m}$ long cable), (v) axial strain (over a section of $10 \mathrm{~cm}$ ) and (vi) temperature (a $10 \mathrm{~m}$ section at a $300 \mathrm{~m}$ long cable was immersed in water of various temperatures). Based on the results, the authors suggested the possibility of discriminating the different types of external perturbation by the specific change in the shapes of the backscattered traces and further detailed investigation will be necessary to correlate the various POF responses to the applied perturbations. The authors argued that if low-cost instrumentation could be developed, the POFOTDR system will be a competitive candidate for short range distributed SHM.

Fukumoto et al. [39] extended the work on POF-OTDR by conducting a feasibility test of the distributed strain sensor for detecting deformation in wooden structures. In their work, the authors were able to detect the direction and magnitude of deformations at four corners of a rectangular wooden frame. The "memory effect" of the POF was also studied and it was reported that when strain is applied to the POF cable, it could be memorised through the plastic deformation of the core material of the POF, and could be read out using the OTDR even after the strain was removed. The spatial resolution of their set-up was reported to be $5 \mathrm{~m}$ for the conventional step-index PMMA POF used (Eska Premier GH-400l-P).

The application of POF using OTDR technology has also been reported elsewhere by Krebber and workers [32, 33, 40]. In their studies, standard SI PMMA POF was integrated into geotextile materials and was shown to be capable of measuring up to $45 \%$ strain. Here, it was observed that the level of the backscattered light increases in a non-linear manner with strain up to $16 \%$ at locations where strain was applied to the POF. The results concur with that reported by Husdi et al. $[37,38]$. Due to the high loss experienced in standard SI PMMA POF $(150 \mathrm{~dB} / \mathrm{km})$, perfluorinated graded-index (GI) POFs (loss of $30 \mathrm{~dB} / \mathrm{km}$ ) were also studied for distributed strain sensing. It was observed that the length of fibre monitored extended from $100 \mathrm{~m}$ for standard PMMA POF to $500 \mathrm{~m}$ in these GI-POFs due to the lower modal dispersion in these graded-index fibres $[32,41]$. For the GIPOFs studied, it was highlighted that the GI-POF tested exhibit a rather nonlinear backscatter increase up to about $3 \%$ strain, above which no further backscatter increase was observed.

In addition to strain monitoring in geotextile materials, the POF was also used for detection of crack opening in masonry structures up to $20 \mathrm{~mm}$ in steps of $2 \mathrm{~mm}$ [32]. Two displacement transducers were used as reference sensors which monitored the width of the crack opening continuously. The results showed that the OTDR backscatter signal increased in respond to widening crack width (up to $25 \mathrm{~mm}$ ) highlighting the feasibility of using POF OTDR sensor to detect cracks in masonry ad concrete structures. The POF sensor was integrated into a geotextile and then surfaced attached to a concrete beam specimen with a small pre-crack. The backscatter signal for two textile specimens was found to increase in a reasonably linear manner (approximately 
$0.05 \mathrm{~dB} / \mathrm{mm}$ crack width) although the authors highlighted that further tests will need to be conducted to obtain reproducibility in the results.

Although the technique above is excellent for monitoring long, large sections of structures/materials, it is primarily suited for quasistatic measurements since data acquisition and processing time of a few seconds to a few minutes are required particularly if high resolution measurement of extended POF length is important to the user. The use of OTDR technique for measurement of strain would only be meaningful if the strain levels are of the order of $1 \%$ and above and a gauge length of tens of centimetre to $1 \mathrm{~m}$. This would be suitable for very large structures with large strains such as the deployment of the POF-OTDR sensor in geotextiles materials as outlined in the review above but may encounter problems if applied to structures with smaller dimension. In addition, the POF OTDR equipment may be prohibitively costly in most situations and hence limited to special niche areas in which their distributed strain monitoring capability is exploited and where their initial investment could be justified.

2.3. Interferometric-Based POF Sensors. Recent progress in the fabrication of single-mode POFs has made possible the use of these fibres for large-strain applications based on interferometric sensing techniques. Here, the principle of operation involves the monitoring of the phase-shift of the propagated light in the test fibre under an applied strain relative to an unstrained reference fibre. The phase-shift is monitored using an interferometric set-up which allows measurement for a limited range of strain values, although it was also reported that using an alternative approach to measuring the phase-shift based on the absolute position time-of-flight telemeter technique has been reported to be useful for strain measurement up to $15.8 \%$ strain in a single-mode PMMA fibre [30]. The high precision and immunity to fluctuation due to light source and bends in the fibre are advantages associated with interferometricbased techniques and since single-mode fibres are smaller in diameters compared to their multi-mode counterparts, they are less intrusive in cases where their embedment could lead to discontinuity in the geometrical build-up, for example, as embedded sensors in composite laminates. On the other hand, however, care and skill are required to successfully cleave and couple these single-mode fibres together, particularly in field environment, to ensure minimal coupling losses in addition to the inherent light loss due to the fibre material itself (typically $150 \mathrm{~dB} / \mathrm{m}$ at $1500 \mathrm{~nm}$ ).

An initial study by Silva-Lopez et al. [42] reported the sensitivity of dye doped single-mode PMMA fibres to strain and temperature using a Mach-Zehnder interferometric setup. The study involved the loading of the fibre on a translation stage where the authors reported the phase sensitivity $\left(1.31 \times 10^{7} \mathrm{rad} / \mathrm{m}\right)$ of the fibre for strain range of $0-0.04 \%$. Kiesel et al. [30] has conducted further experiments using single-mode POF where the fibres were tested for their strain response to failure in order to determine the calibration coefficients at strain rates from $0.01 / \mathrm{min}$ to $3.05 / \mathrm{min}$. The typical failure strains of the POF specimens used was $30 \%$. They reported an upper limit of fringe visibility at $15.8 \%$ nominal strain in the fibres used indicating the maximum strain possible for the POF tested using the set-up in their study. The calibrated linear and nonlinear coefficients were found to be $1.37 \times 10^{7} \mathrm{rad} / \mathrm{m}$ and $3.1 \times 10^{6} \mathrm{rad} / \mathrm{m}$, respectively.

Strain measurement based on the fibre stretching of $1 \mathrm{~mm}$ diameter multi-mode PMMA POF was demonstrated recently by Poisel [43]. The author monitored the phase shift of a sinusoidal signal in the fibre under various tensile loads (corresponding to increasing extension of the POF from 0 to $500 \mu \mathrm{m}$ in steps of $50 \mu \mathrm{m}$ ). The interrogation set-up relies on detecting the difference in transit times through the polymer optical fibre (POF) using an electronic phase-shift detector. A resolution of $10 \mu \mathrm{m}$ extension was reported to be possible under a tensile set-up. The simple system was also shown to be sensitive to bending loads and capable of measuring dynamic loading up to $5 \mathrm{~Hz}$.

These studies have demonstrated the potential of interrogating a section of stretched fibre for strain monitoring. However, there is still limited work to demonstrate the higher dynamic strain measurement capability of the technique (which could be applied for SHM applications). In addition, interferometric sensing in general requires a stable platform due to their susceptibility to vibration-induced noise and hence further work will be required before this approach to POF strain sensing can be applied in real structures.

2.4. Other POF-Based Sensors for SHM Applications. Fibre Bragg grating (FBG) sensors are well known for their capability for strain measurement and the gratings are typically inscribed in silica optical fibres, the fundamentals of which are well documented elsewhere [47, 48]. Briefly, it involves monitoring the shift in the peak or resonance wavelength of either the reflected or transmitted spectrum resulting from an applied strain on the fibre. The possibility of absolute strain measurement and multiplexing capability of grating-based optical fibre sensors has received considerable attention for application in structural health monitoring. For SHM applications, FBG has featured extensively in many published articles for monitoring a variety of physical parameters $[1,2]$. Monitoring of strain in this class of fibre sensors relies on detecting the shift in the central wavelength of the reflection spectrum as a response to applied strain.

The ability to induce a periodic refractive-index change in polymer-based optical fibres is a recent development and this has opened up further SHM applications using gratingbased sensors since the availability of POF-based FBG sensors offers ease of handling, higher strain sensitivity $(1.48 \mathrm{pm} / \mu \varepsilon$ compared to $1.15 \mathrm{pm} / \mu \varepsilon$ for silica-based FBGs at wavelength of $1523 \mathrm{~nm}$ ) and higher strain limit (up to $3.61 \%$ strain compared to $0.5-0.6 \%$ strain for silica-based FBGs) [17-19, $45,46]$. Although more work and attention are required to further improve and understand the grating writing process in polymer fibres, their potential for SHM is evident. The possibility of using a POF-based FBG sensor for strain and temperature measurement have been reported and shown to allow strain monitoring up to seven times the measurement 
limit of its silica counterpart $[45,46]$. The sensor used exhibited good reproducibility and reversibility over the large strain sensing range. In their studies, the authors showed that the polymer FBG sensor was able to sustain up to $3 \%$ strain before yielding and by using a combination of polymer and silica FBG, it was possible to discriminate between the effect of temperature and strain on the sensor read-out [46]. Using a matrix inversion technique and solving for change in temperature, $\Delta T=f\left\{\Delta \lambda_{\text {POF-FBG }}\right\}$ and change in strain, $\Delta \varepsilon=f\left\{\Delta \lambda_{\text {Silica-FBG }}\right\}$ which are formulated using the strain and temperature sensitivities of the POF-FBG and the silicaFBG, respectively, the technique allows the applied strain and temperature to be determined simultaneously.

As a response to the lack of commercial availability of suitable single-mode POF, Krebber et al. [44] demonstrated the use of a tapered multimode standard POF and nontapered GI-POF for creating grating-based sensors. The authors successfully generated the gratings in the tapered POF (from original $0.75 \mathrm{~mm}$ fibre to $0.2 \mathrm{~mm}$ ) and nontapered GI-POF specimens which showed strong reflection spectrum at specific locations along the POF using an OTDR technique and reported that it was possible to measure the integral strain along the fibre and to resolve the local strain at various locations.

Writing long period gratings (LPGs) in POFs has been reported recently [19] although there appears to be little published work in the literature on their application for strain sensing. Li et al. reported success of inducing gratings in a highly photosensitive POF core using traditional photoetching technology together with a low-cost high pressure mercury lamp as the light source but no mechanical test was carried to assess the mechanical response of the sensor produced. The transmission spectrum of the written LPG demonstrated a loss of $3 \mathrm{~dB}$ at a peak wavelength of $1568 \mathrm{~nm}$; despite PMMA having peak transmission in the visible spectrum, presumably to interface with optical telecommunications spectrometers and lightsources using with glass optical fibres. For Bragg gratings in the visible spectrum, the required periodicity of refractive index gratings is 183 to $216 \mathrm{~nm}$; for long period gratings, the periodicity is 100 to $500 \mu \mathrm{m}$ so it can be easily created by direct laser writing. One reason for the lack of published work is due to short supply of good quality commercially available singlemode POF with low loss and doped for photosensitivity, another is the lack of availability of single mode POF connectors since the fibre is produced in non standard diameters.

One of the most recent developments in polymer fibre research which has attracted significant attention is the microstructured POF (mPOF). This type of POF is easy to manufacture with consistent quality, but is not commercially available at present, although it may be available for research groups who are keen to find applications for their mPOFs. Interestingly LPGs have also been fabricated on mPOFs and tested for their mechanical response [49]. MPOF is ideal for FBG and LPG as it is easy to handle, since the mPOF has a large diameter and yet is endlessly singlemoded along its length with low loss compared with single mode POF. In mPOF, the microscopic air channels that run along the length of the fibre defines the light guiding mechanism in contrast to the variations in the refractive index of the fibre material in conventional POF [21]. Two of the main advantages of using LPGs in mPOFs over standard PMMA fibres include the possibility of tuning the sensitivities of the loss features corresponding to the different measurands of interest by altering the hole geometry in mPOFs [21] and the possibility of introducing directional bend sensitivity [24]. LPG has been introduced in mPOFs by mechanical imprinting using a $15 \mathrm{~cm}$ long template (with period of $1 \mathrm{~mm}$ ) placed upon a heated fibre and in their study, Large et al. [25] studied the viscoelastic properties of the mPOF and reported that shift in spectral features for strains up to $8 \%$ was possible although above $2 \%$ strain a non-linear response was reported to be evident. Below 2\% strain, the change in peak wavelength to strain could be computed to be approximately $1.2 \mathrm{pm} / \mu \varepsilon$. The study also showed a nonreversible deformation response due to strain-related creep following a 10 hour constant strain at 3\% strain. In addition, the authors also reported a wavelength shift of up to $4 \mathrm{~nm}$ due to material relaxation at strains of $6 \%$. In cases where intermittent straining was applied on the MPOF LPG sensor for up to $2 \%$ strain, the authors argued that the viscoelastic effects (time, strain rate, and strain magnitude) were small although time-dependent effects such as relaxation during constant strain and strain recovery could become significant. In SHM applications where these limitations are properly understood and deemed acceptable, mPOF LPG sensors may be successfully applied.

Conventionally, fibre Bragg gratings and long-period gratings sensors are interrogated using an optical spectrum analyser to detect the shift in wavelength of the reflected spectrum corresponding to the applied strain and/or temperature. However, the associated interrogation equipment is generally costly, involving the use of optical spectrum analysers and narrow band light sources such as laser diode. In an effort to circumvent the cost barrier to the wider adoption of grating-based sensors, Hwang et al. [49] recently demonstrated an interesting intensity-based set-up which utilizes two long-period fibre gratings and a core mode blocker between the two gratings. Although the fibers used were not polymer-based, this work is highlighted in view of the potential of the technique to be applied for interrogation of POF-based grating sensors. In principle, the core mode blocker acts as a band-pass filter to block the uncoupled light while the light that satisfy the phase-matching condition of the first LPG will be coupled to the cladding mode. The light in the cladding layer would then be effectively coupled back into the core by the second LPG. Strain is then applied on one of the LPGs. The relative change in the resonance wavelengths (change in the degree of spectrum overlap) due to the strain applied on one of the LPGs will result in either increase or decrease in the transmitted optical power. The power is monitored and then calibrated against the applied strain. In their work, the authors were able to show that the intensity transmitted through the fibre increases linearly with strain although the strain sensitivity $\left(1.0 \times 10^{-4} \mathrm{~dB} / \mu \varepsilon\right)$ was noted to be three time less than conventional single long-period grating fibre sensor [50]. The authors also noted small fluctuation in the transmitted 


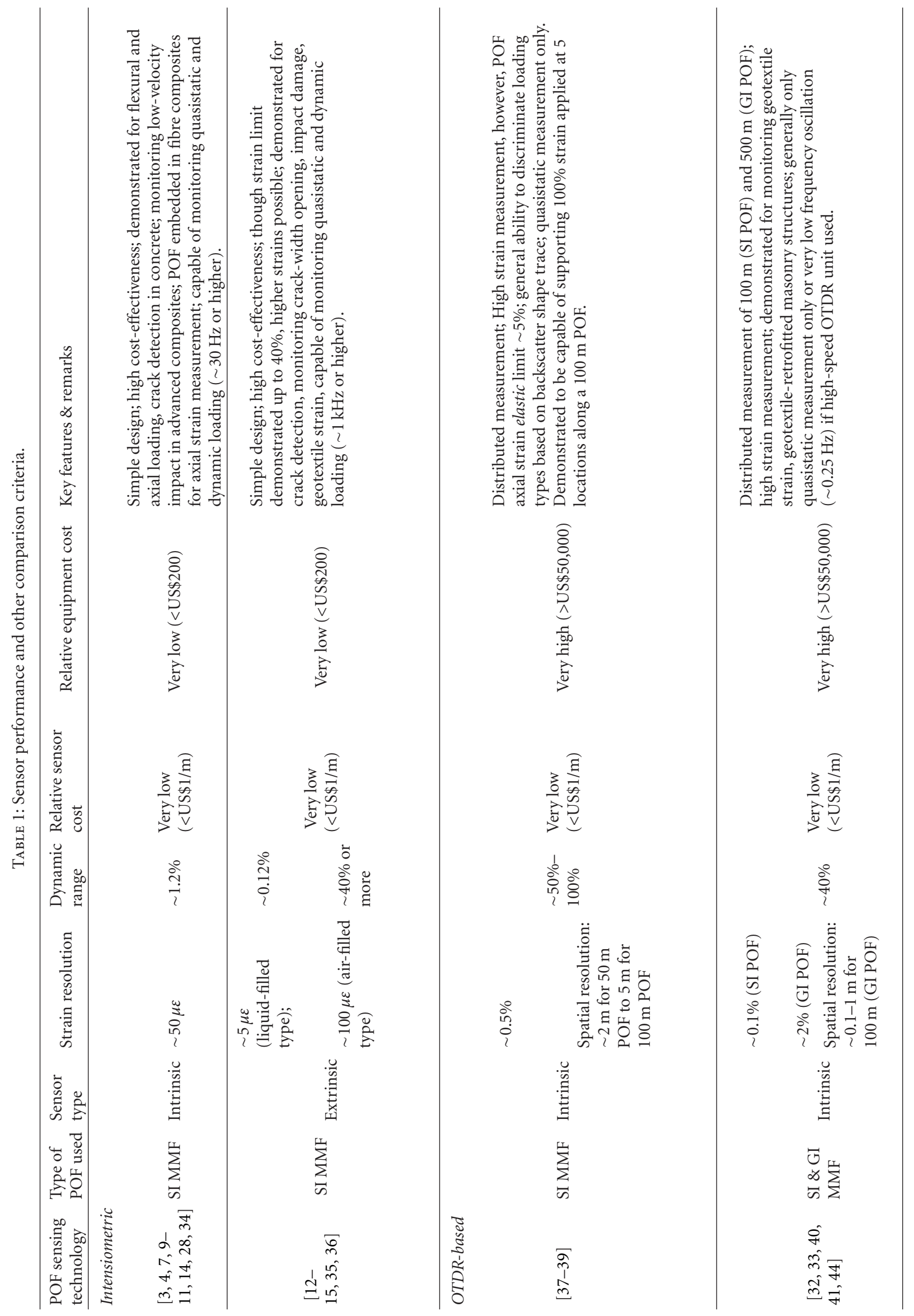




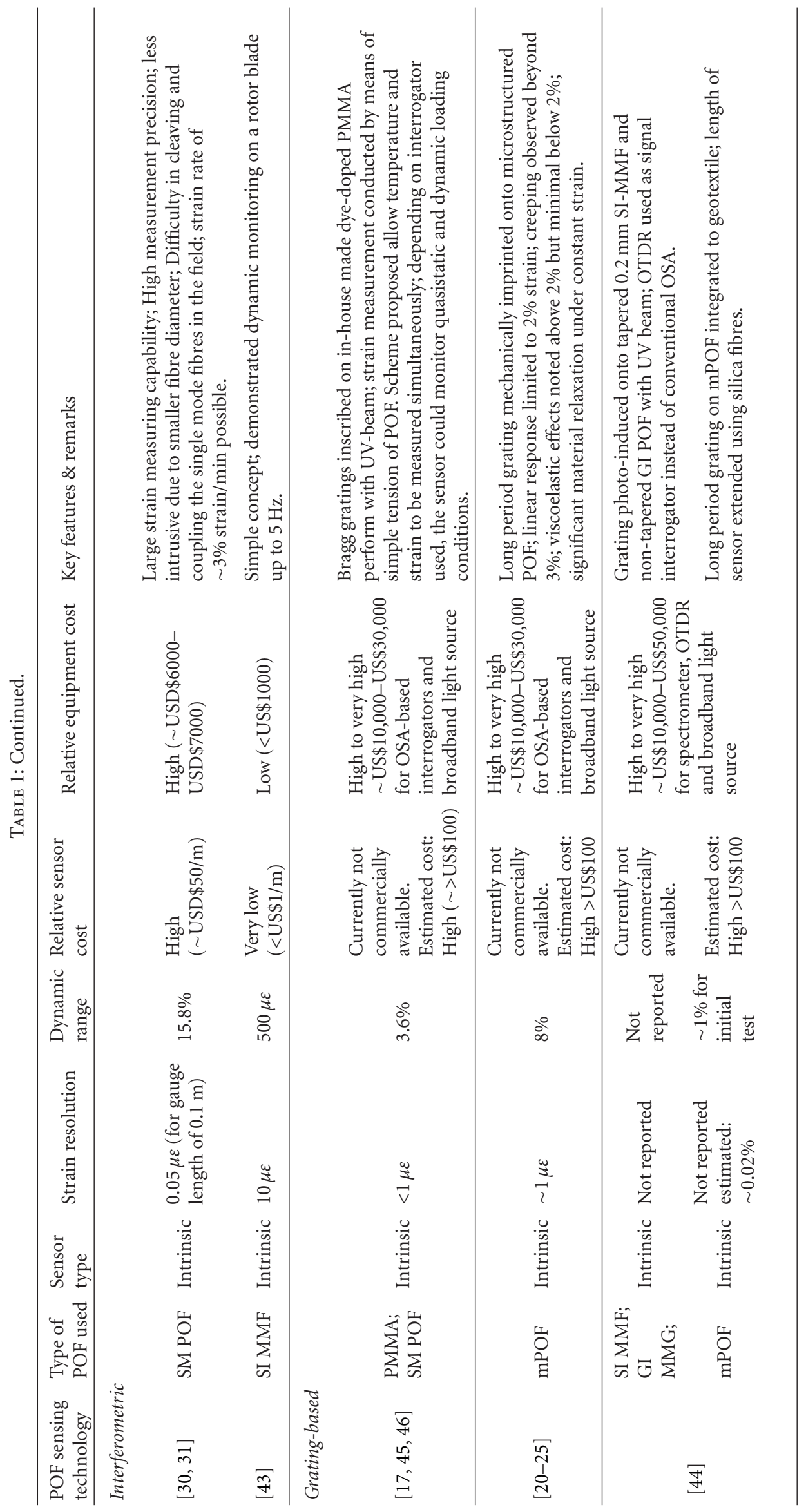


power resulting from the unstable LED source though it was unclear whether the observed fluctuation affected the accuracy of the measurements significantly. Despite the lower sensitivity of the system (thought to be due to the broader bandwidth of the LPG relative to a laser diode), small fluctuation in transmitted power, the authors argued that the cost-effectiveness of the proposed sensor system would see its application for interrogation of grating-based sensors. With the possibility of introducing gratings in POFs as outlined by the reports above, it would be interesting to see further developments and field applications in intensity-based POF grating sensors for SHM applications using the technique proposed.

\section{Summary and Conclusions}

An overview of a selection of articles on the recent progress of the applications of plastic-based optical fibre sensors has been given. A tabulated summary highlighting the major works and a brief comparison of the types of sensors reviewed is shown in Table 1 . The overview began with a brief introduction of the technology and sensing techniques used in POF sensors highlighting the various strengths and limitations associated with the different sensing schemes adopted. In view of the ease of handling, low cost and large strain capability of POF, significant interest in their use for structural health monitoring, specifically for measurement of strain, curvature, load, displacement, vibration and crack detection have been demonstrated by various workers. A number of different approaches in sensing interrogation such as intensity-based, interferometric-based, time-of-flight (OTDR-based) and gratings (wavelength-based) have been shown to be feasible for monitoring engineering materials such as concrete, masonry, fibre composites, geotextiles, metals, wood and plastics. There remains much work to be done to fully characterize the POF response to the various physical measurands encountered in SHM applications particularly with the recent development of new POF materials, types and interrogation techniques.

\section{Acknowledgments}

Funding by A*STAR-MPA through the National University of Singapore CORE centre under joint Grants no. R-264-000226-490 and no. R-264-000-226-305 is acknowledged.

\section{References}

[1] K. S. C. Kuang and W. J. Cantwell, "The use of conventional optical fibres and fibre Bragg gratings for damage detection in advanced composite structures—a review," Applied Mechanics Reviews, vol. 56, pp. 493-513, 2003.

[2] G. Zhou and L. M. Sim, "Damage detection and assessment in fibre-reinforced composite structures with embedded fibre optic sensors-review," Smart Materials and Structures, vol. 11, no. 6, pp. 925-939, 2002.

[3] K. S. C. Kuang, W. J. Cantwell, and P. J. Scully, "An evaluation of a novel plastic optical fibre sensor for axial strain and bend measurements," Measurement Science and Technology, vol. 13, no. 10, pp. 1523-1534, 2002.
[4] A. Babchenko, Z. Weinberger, N. Itzkovich, and J. Maryles, "Plastic optical fibre with structural imperfections as a displacement sensor," Measurement Science and Technology, vol. 17, no. 5, pp. 1157-1161, 2006.

[5] N. Takeda, T. Kosaka, and T. Ichiyama, "Detection of transverse cracks by embedded plastic optical fiber in FRP laminates," in Smart Structures and Materials 1999: Sensory Phenomena and Measurement, vol. 3670 of Proceedings of SPIE, pp. 248-255, 1999.

[6] N. Takeda, "Characterization of microscopic damage in composite laminates and real-time monitoring by embedded optical fiber sensors," International Journal of Fatigue, vol. 24, no. 2-4, pp. 281-289, 2002.

[7] A. Djordjevich, "Curvature gauge as torsional and axial load sensor," Sensors and Actuators A, vol. 64, no. 3, pp. 219-224, 1998.

[8] G. Perrone, D. Perla, R. Gaudino, and S. Abrate, "Development of low cost intensity modulated POF based sensors and application to the monitoring of civil structures," in Proceedings of the 13th Plastic Optical Fibre Conference, pp. 444-449, 2004.

[9] K. S. C. Kuang and W. J. Cantwell, "The use of plastic optical fibres and shape memory alloys for damage assessment and damping control in composite materials," Measurement Science and Technology, vol. 14, no. 8, pp. 1305-1313, 2003.

[10] K. S. C. Kuang and W. J. Cantwell, "The use of plastic optical fibre sensors for monitoring the dynamic response of fibre composite beams," Measurement Science and Technology, vol. 14, no. 6, pp. 736-745, 2003.

[11] K. S. C. Kuang, Akmaluddin, W. J. Cantwell, and C. Thomas, "Crack detection and vertical deflection monitoring in concrete beams using plastic optical fibre sensors," Measurement Science and Technology, vol. 14, no. 2, pp. 205-216, 2003.

[12] K. S. C. Kuang, M. Maalej, and S. T. Quek, "An application of a plastic optical fiber sensor and genetic algorithm for structural health monitoring," Journal of Intelligent Material Systems and Structures, vol. 17, no. 5, pp. 361-379, 2006.

[13] K. S. C. Kuang, S. T. Quek, and M. Maalej, "Detection of impact induce damage in composite beams using plastic optical fibre sensors," in Proceedings of the 17th KKCNN Symposium on Civil Engineering, pp. 103-110, 2004.

[14] K. S. C. Kuang, S. T. Quek, and M. Maalej, "Polymer-based optical fiber sensors for health monitoring of engineering structures," in Smart Structures and Materials 2005: Sensors and Smart Structures, vol. 5765 of Proceedings of SPIE, pp. 656667, 2005.

[15] K. S. C. Kuang, S. T. Quek, and M. Maalej, "Assessment of an extrinsic polymer-based optical fibre sensor for structural health monitoring," Measurement Science and Technology, vol. 15, no. 10, pp. 2133-2141, 2004.

[16] K. S. C. Kuang, S. T. Quek, and W. J. Cantwell, "Use of polymer-based sensors for monitoring the static and dynamic response of a cantilever composite beam," Journal of Materials Science, vol. 39, no. 11, pp. 3839-3843, 2004.

[17] P. J. Scully, D. Jones, and D. A. Jaroszynski, "Femtosecond laser irradiation of polymethylmethacrylate for refractive index gratings," Journal of Optics A, vol. 5, no. 4, pp. S92-S96, 2003.

[18] G. D. Peng and P. L. Chu, "Polymer optical fiber sensing," in Optical Information Processing Technology, vol. 4929 of Proceedings of SPIE, pp. 303-311, 2002.

[19] Z. Li, H. Y. Tam, L. Xu, and Q. Zhang, "Fabrication of longperiod gratings in poly(methyl methacrylate-co-methyl vinyl ketone-co-benzyl methacrylate)-core polymer optical fiber by use of a mercury lamp," Optics Letters, vol. 30, no. 10, pp. 1117-1119, 2005. 
[20] M. C. J. Large, L. Poladian, G. W. Barton, and M. A. van Eijkelenborg, Microstructured Polymer Optical Fibres, Springer, New York, NY, USA, 2007.

[21] M. A. van Eijkelenborg, M. C. J. Large, A. Argyros, et al., "Microstructured polymer optical fiber," Optics Express, vol. 9, pp. 319-327, 2001.

[22] H. Dobb, D. J. Webb, K. Kalli, A. Argyros, M. C. J. Large, and M. A. van Eijkelenborg, "Continuous wave ultraviolet light-induced fibre Bragg gratings in few- and single-moded microstructured polymer optical fibres," Optical Letters, vol. 30, pp. 3296-3298, 2005.

[23] M. P. Hiscocks, M. A. van Eijkelenborg, A. Argyros, and M. C. J. Large, "Stable imprinting of long-period gratings in microstructured polymer optical fibre," Optics Express, vol. 14, no. 11, pp. 4644-4649, 2006.

[24] H. Dobb, K. Kalli, and D. J. Webb, "Measured sensitivity of arcinduced long-period grating sensors in photonic crystal fibre," Optics Communications, vol. 260, no. 1, pp. 184-191, 2006.

[25] M. C. J. Large, J. Moran, and L. Ye, "The role of viscoelastic properties in strain testing using microstructured polymer optical fibres (mPOF)," Measurement Science and Technology, vol. 20, no. 3, Article ID 034014, 6 pages, 2009.

[26] K. Murphy, S. Zhang, and V. M. Karbhari, "Effect of concrete based alkaline solutions on short term response of composites," in Proceedings of the 44th International Society for the Advancement of Material and Process Engineering Symposium and Exhibition, vol. 44, pp. 2222-2230, 1999.

[27] D. Kalymnios, P. J. Scully, J. Zubia, and H. Poisel, "POF sensor overview," in Proceedings of the 13th International Plastic Optical Fibres Conference, pp. 237-244, 2004.

[28] Y. M. Wong, P. J. Scully, R. J. Bartlett, K. S. C. Kuang, and W. J. Cantwell, "Plastic optical fibre sensors for environmental monitoring: biofouling and strain applications," Strain, vol. 39, no. 3, pp. 115-119, 2003.

[29] R. J. Bartlett, R. Philip-Chandy, P. Eldridge, D. F. Merchant, R. Morgan, and P. J. Scully, "Plastic optical fiber sensors and devices," Transactions of the Institute of Measurement and Control, vol. 22, pp. 431-457, 2000.

[30] S. Kiesel, K. Peters, T. Hassan, and M. Kowalsky, "Calibration of a single-mode polymer optical fiber large-strain sensor," Measurement Science and Technology, vol. 20, no. 3, Article ID 034016, 7 pages, 2009.

[31] S. Kiesel, K. Peters, T. Hassan, and M. Kowalsky, "Behaviour of intrinsic polymer optical fibre sensor for large-strain applications," Measurement Science and Technology, vol. 18, no. 10, pp. 3144-3154, 2007.

[32] S. Liehr, P. Lenke, K. Krebber, et al., "Distributed strain measurement with polymer optical fibers integrated into multifunctional geotextiles," in Optical Sensors 2008, vol. 7003 of Proceedings of SPIE, April 2008, article no. 700302.

[33] P. Lenke, S. Liehr, K. Krebber, F. Weigand, and E. Thiele, "Distributed strain measurement with polymer optical fiber integrated in technical textiles using the optical time domain reflectometry technique," in Proceedings of the 16th International Conference on Plastic Optical Fibre, pp. 21-24, 2007.

[34] P. Cortes, W. J. Cantwell, K. S. C. Kuang, and S. T. Quek, "The morphing properties of a smart fibre metal laminate," Polymer Composites, vol. 29, pp. 1263-1268, 2008.

[35] K. S. C. Kuang, S. T. Quek, and W. J. Cantwell, "Morphing and control of a smart fibre metal laminate utilizing plastic optical fibre sensor and Ni-Ti sheet," in Proceedings of the 17th International Conference on Composite Materials, 2009.
[36] K. S. C. Kuang, S. T. Quek, C. Y. Tan, and S. H. Chew, "Plastic optical fiber sensors for measurement of large strain in geotextile materials," Advanced Materials Research, vol. 4750, pp. 1233-1236, 2008.

[37] I. R. Husdi, K. Nakamura, and S. Ueha, "Sensing characteristics of plastic optical fibres measured by optical time-domain reflectometry," Measurement Science and Technology, vol. 15, no. 8, pp. 1553-1559, 2004.

[38] K. Nakamura, I. R. Husdi, and S. Ueha, "Memory effect of POF distributed strain sensor," in Second European Workshop on Optical Fibre Sensors, vol. 5502 of Proceedings of SPIE, pp. 144-147, 2004.

[39] T. Fukumoto, K. Nakamura, and S. Ueha, "A POF-based distributed strain sensor for detecting deformation of wooden structures," in 19th International Conference on Optical Fibre Sensors, vol. 7004 of Proceedings of SPIE, 2008, article no. 700469.

[40] K. Krebber, P. Lenke, S. Liehr, J. Witt, and M. Schukar, "Smart technical textiles with integrated POF sensors," in Smart Sensor Phenomena, Technology, Networks, and Systems 2008, vol. 6933 of Proceedings of SPIE, 2008, article no. 69330V.

[41] P. Lenke, S. Liehr, K. Krebber, F. Weigand, and E. Thiele, "Distributed strain measurement with polymer optical fiber integrated in technical textiles using the optical time domain reflectometry technique," in Proceedings of the 16th International Conference on Plastic Optical Fibre, pp. 21-24, 2007.

[42] M. Silva-Lopez, A. Fender, W. N. MacPherson, et al., "Strain and temperature sensitivity of a singlemode polymer optical fibre," Optics Letters, vol. 30, pp. 3129-3131, 2005.

[43] H. Poisel, "POF strain sensor using phase measurement techniques," in Smart Sensor Phenomena, Technology, Networks, and Systems 2008, vol. 6933 of Proceedings of SPIE, 2008, article no. 69330 Y.

[44] K. Krebber, P. Lenke, S. Liehr, et al., "Technology and applications of smart technical textiles based on POF," in Proceedings of the 17th International Conference on Plastic Optical Fibre, 2008.

[45] H. Y. Liu, H. B. Liu, and G. D. Peng, "Strain sensing characterization of polymer optical fibre Bragg gratings," in 17th International Conference on Optical Fibre Sensors, vol. 5855 of Proceedings of SPIE, pp. 663-666, 2005.

[46] H. B. Liu, H. Y. Liu, G. D. Peng, and P. L. Chu, "Strain and temperature sensor using a combination of polymer and silica fibre Bragg gratings," Optics Communications, vol. 219, no. 16, pp. 139-142, 2003.

[47] K. O. Hill, Y. Fuji, D. C. Johnson, and B. S. Kawasaki, "Photosensitivity in optical fiber waveguides, application to reflection filter fabrication," Applied Physics Letters, vol. 32, pp. 647-649, 1978.

[48] G. Meltz, G. G. Morey, and W. H. Glenn, "Formation of Bragg grating in optical fibers by a transverse holographic method," Optics Letters, vol. 14, pp. 823-825, 1989.

[49] D. Hwang, L. V. Nguyen, D. S. Moon, and Y. J. Chung, "Intensity-based optical fiber strain sensor," Measurement Science and Technology, vol. 20, Article ID 034020, 6 pages, 2009.

[50] M. de Vries, V. Bhatia, T. D’Alberto, V. Arya, and R. O, Claus, "Photoinduced grating-based optical fiber sensors for structural analysis and control," Engineering Structures, vol. 20, no. 3, pp. 205-210, 1998. 

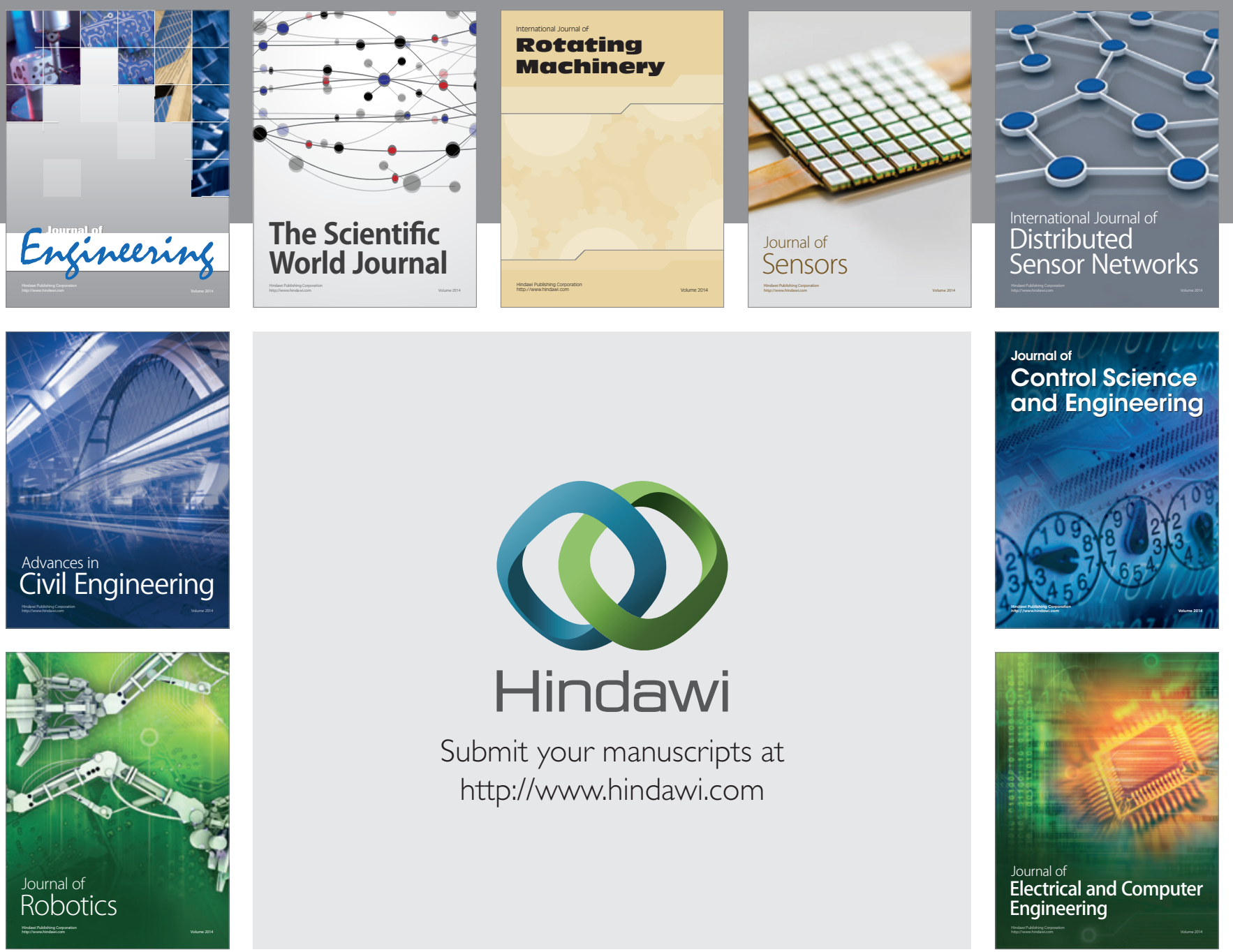

Submit your manuscripts at

http://www.hindawi.com
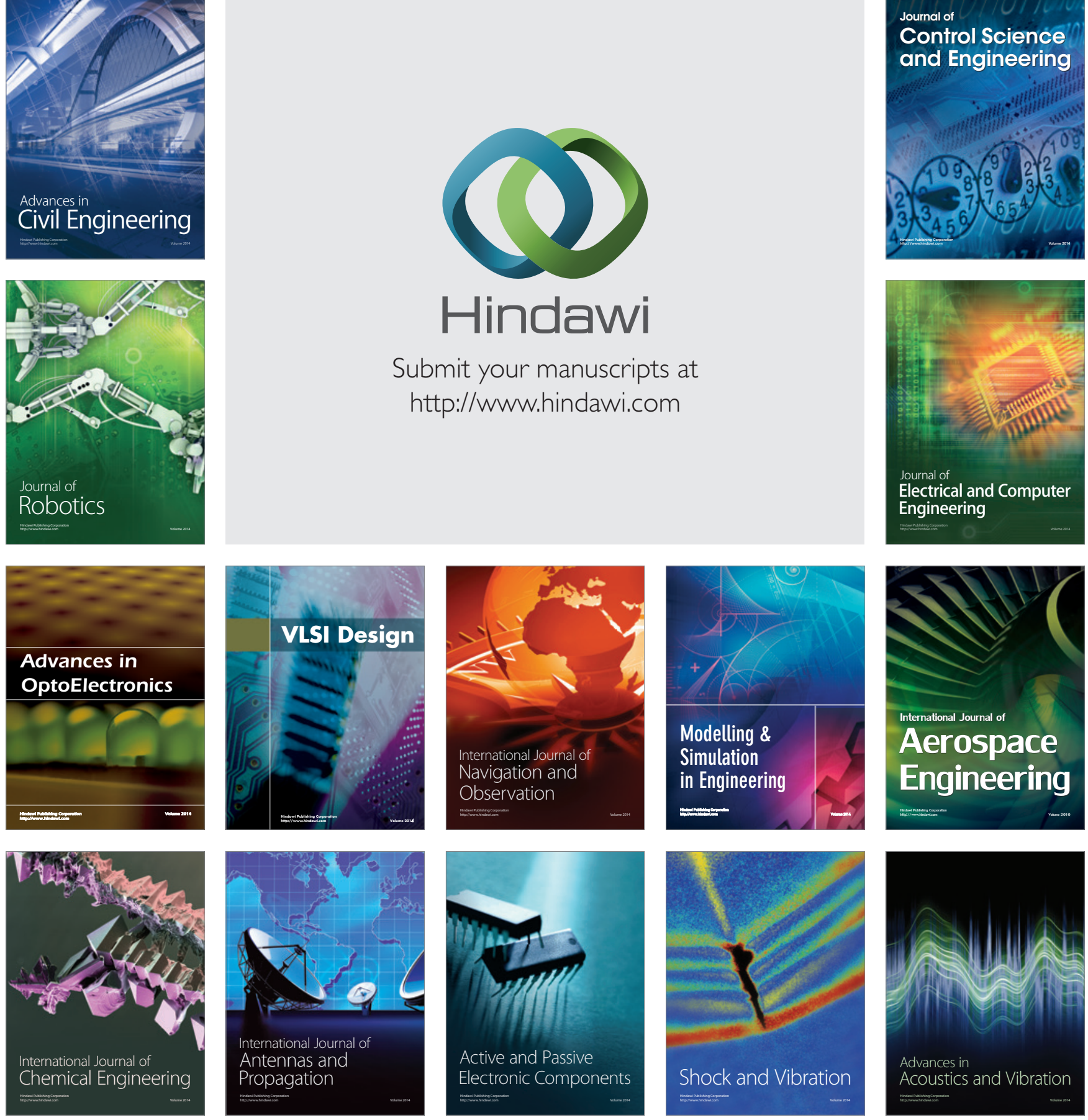